\title{
The golden hour of acute ischemic stroke
}

\author{
Rajiv Advani ${ }^{1,2^{*}}$, Halvor Naess ${ }^{3,4}$ and Martin W. Kurz ${ }^{1,2}$
}

\begin{abstract}
Background: Acute Ischemic Stroke (AIS) treatment has been revolutionised in the last two decades with the increasing use of Intravenous Thrombolysis (IVT) and with the advent of Endovascular therapy (EVT). AlS treatment and outcome are time dependant and time saving measures are being implemented at every step of the treatment chain. These changes have resulted in lower treatment times in-hospital, but it is unclear if this translates into more patients being treated within 60 min of symptom onset - the Golden Hour. The clinical outcome of IVT therapy in this patient group was our secondary outcome.
\end{abstract}

Methods: From 2009 onwards, systematic changes were made to the AlS treatment chain leading to a dramatic decrease in Door-to-Needle (DTN) time. Analyses were performed on the number of these treatments year on year and their clinical outcomes within the Golden Hour at Stavanger University Hospital (SUS).

Results: Six-hundred and thirteen patients were included; seventy-three were treated within the Golden Hour. The percentage of total IVT treatments occurring in the Golden Hour rose from 2.2\% in 2009 to $14.5 \%$ in 2015 ( $p=0.006$ ) with a high of $18.3 \%$ in $2012(p<0.001)$. All of these patients had a Median NIHSS of 0 at discharge, irrespective of age and pre-existing comorbidity. There was no incidence of any $\mathrm{ICH}$ and in-hospital mortality was only $2.7 \%$ in this group.

Discussion: The time from AIS symptom onset to treatment is filled with delays. Despite the inherence of some delays,significant efforts on the part of the pre- and in- hospital treatment chain have made IVT therapy within 60 min a possibility. The allocation and use of resources in the setting of rapid AIS treatment is warrantedand yields unprecedented results.

Conclusions: Our study shows that improved treatment routines led to an increase in the number of patients treated within the Golden Hour. Treatment in the Golden Hour leads to excellent outcomes in all patients, irrespective of age and pre-existing comorbidity.

\section{Background}

The last two decades have seen a revolution in the treatment of acute ischemic stroke (AIS), initially with the use of intravenous thrombolysis (IVT) [1-3] and more recently with the advent of endovascular thrombectomy (EVT) [4-6]. An increasing number of patients have had access to therapy over recent years due to improvements in pre- and intra- hospital systems $[7,8]$. However, both these therapeutic modalities are time dependant and analyses have shown that better outcomes

\footnotetext{
* Correspondence: advanirajiv@gmail.com

${ }^{1}$ Department of Neurology, Stavanger University Hospital, Postboks 8100, Stavanger 4068, Norway

${ }^{2}$ Neuroscience Research Group, Stavanger University Hospital, Stavanger, Norway

Full list of author information is available at the end of the article
}

can be expected when treatment is administered sooner rather than later within the therapeutic window [9-11].

Recent analyses into outcomes of treatment have shown that treatment within $60 \mathrm{~min}$ of symptom onset produces excellent outcomes with significantly lower rates of morbidity and mortality in younger patients [12]. The authors suggest that the results are so encouraging that pre-hospital IVT should be the way forward in AIS treatment. This remarkable 60 min window from the onset of symptoms has been increasingly known as The Golden Hour.

However, IVT therapy in the pre-hospital setting is fraught with challenges, a recently published editorial highlights these challenges and errs on the side of caution [13].

At our treatment centre, Stavanger University Hospital (SUS), we have worked systematically since 2009 to 
reduce the time used in-hospital to treat patients with AIS. The aim of these changes was to reduce the Doorto-Needle (DTN) time. The successfully implemented organisational changes, described in detail in earlier publications [7, 14], reduced the median DTN time to under $30 \mathrm{~min}$. Our results also showed that transport times to SUS remained unchanged in the period of our study.

Reducing DTN time shows increased efficacy on the part of the AIS treatment chain, but does this translate into a reduction in Onset-to-Needle (OTN) time - do we treat more patients within The Golden Hour?

Our primary aim was to assess if the falling DTN time was associated with an increase in the number of patients with an OTN time of 60 min or less. Our secondary aims were to assess the clinical outcomes these patients had to see if early treatment correlated with good outcome in our patient population.

\section{Methods}

\section{Patient population/catchment area}

SUS serves a population of almost 350 000, receiving all AIS patients in its catchment area. Approximately 200000 inhabitants live in and around the city of Stavanger; the remainder live in more rural areas of Rogaland County.

All IVT treatments at SUS during the 2009 to 2015 $(n=613)$ are included, irrespective of age, sex, other comorbidities and stroke severity on admission. According to treatment guidelines IVT was administered within 4.5 $h$ of AIS symptom onset. These numbers are inclusive of any stroke mimic diagnoses (both medical and functional) that presented as AIS on admission.

\section{Stroke database}

All patients treated with IVT were retrospectively compiled into a database until 2012, when prospective inclusion began in our stroke database. This database included details from medical records including AIS risk factors, National Institutes of Health Stroke Scale (NIHSS) scored on admission, after IVT therapy and at discharge; treatment complications and in-hospital mortality were also noted. All the applicable times including; time of stroke onset, when the EMS were contacted, the time of admission to the hospital and time of IVT administration were taken from a combination of the prehospital records (AMIS - Acute Medical Information System) and hospital medical records. The relevant time durations including DTN and OTN time were then calculated.

All these data were compiled into a single database.

\section{Clinical follow-up}

Patients with suspected AIS were admitted directly to the ER, where clinical examination to assess NIHSS was performed followed by a head CT (Computed Tomography). Once contraindications had been excluded using the head CT, IVT treatment was administered. Following the IVT bolus, a CT angiography and CT perfusion scan were also performed to determine the presence of a Large Vessel Occlusion (LVO). Patients with a LVO were transferred to the EVT lab and excluded from our analyses. After the administration of IVT, patients were admitted to a neuro-intensive unit for further clinical follow up. NIHSS scoring was performed two hours after IVT administration to assess the therapeutic effect and again at twenty-four hours. A follow-up head CT was performed between 12 and $24 \mathrm{~h}$ after IVT administration to determine the presence of any intracerebral hemorrhage (ICH). In patients with a positive clinical course no further imaging was performed and a final NIHSS assessment was performed on discharge. In those worsening or not showing clinical improvement, cerebral imaging was repeated along with NIHSS assessment at regular intervals. Stroke physicians were in-charge of patient follow up at the ward and the other relevant medical specialties were consulted in conjunction with any complications, i.e. myocardial infarction, infections, etc.

\section{Data analysis}

The afore mentioned database was used to calculate certain key variables such as the DTN time, OTN time, NIHSS on admission and discharge. Using these variables we could ascertain the number of patients treated within the Golden Hour (OTN $\leq 60 \mathrm{~min}$ ) year on year from 2009 up to and including 2015. We could then calculate the percentage of all IVT treatments occurring within $60 \mathrm{~min}$ of onset year by year. Using the NIHSS on admission and discharge we could ascertain whether patients treated within the Golden Hour improved after therapy and if they had neurological deficits if any. The diagnosis at discharge for each patient was recorded in the database so that we could keep track of stroke mimics. Any ICH and or in-hospital mortality was also recorded.

\section{Statistics}

Using the Stroke research database, the data for the above mentioned end points was collected and analyzed. Statistical analyses were conducted to determine any changes in the number of EMS admissions as well as the number of treatments with IVT. Statistical analysis was conducted using IBM SPSS version 22. $P$ values were determined using one-way analysis of variance (ANOVA) and Pearson's Chi squared test as appropriate. $P$ values less than 0.05 were considered significant. 


\section{Percentage of IVT treatments within the Golden Hour}

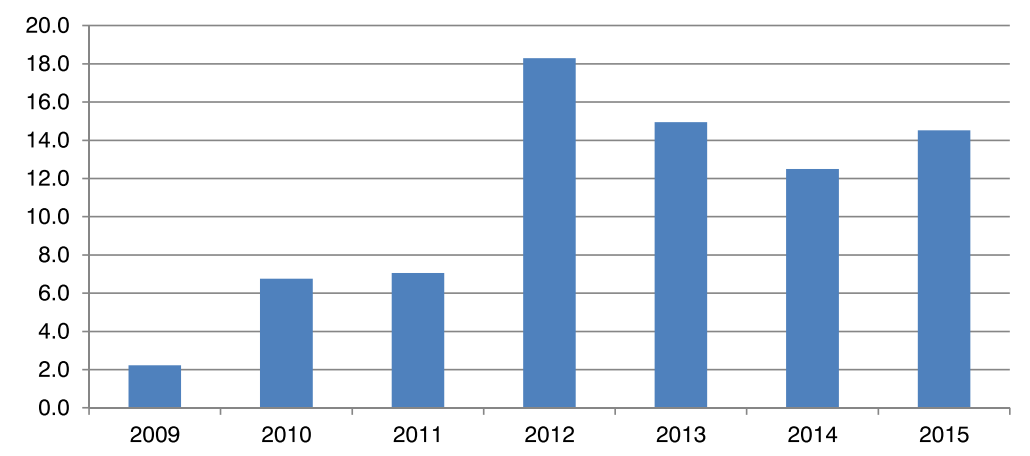

Fig. 1 The percentage of all IVT treatments occurring within the Golden Hour from 2009 - 2015

\section{Results}

Figure 1 shows the percentage of all IVT treatments with an OTN time of less than or equal to $60 \mathrm{~min}$ - i.e. within the Golden Hour. There is a rise in the percentage of patients treated within the Golden Hour from $2.2 \%$ in 2009 to $6.8 \%$ in 2010 and then $7.1 \%$ in 2011 . In 2012 there is a dramatic increase in the percentage treated within $60 \mathrm{~min}$ of onset $-18.2 \%$ of all IVT treatments $(p<0.005)$. This number then falls to $14.9 \%$ in 2013 and to $12.5 \%$ in 2014 before rising to $14.5 \%$ in $2015(p=0.006)$.

Table 1 shows patients treated with IVT divided into the respective therapeutic windows. The first group comprises those treated within the Golden Hour ( $n=$ $73)$, the second group are those treated from $61 \mathrm{~min}$ up to and including $180 \mathrm{~min}(n=414)$ and the final group are those receiving treatment from $181 \mathrm{~min}$ up to and including $270 \mathrm{~min}(n=126)$ after symptom onset. The median age (range) of patients in the three groups was 71 (38-91), 72 (41-93) and 75 (42-94) respectively. Forty-one percent of the Golden Hour group suffered from hypertension, compared to Forty-six percent in the group treated between 61 and 180 min of onset and Fifty-seven percent amongst those treated between 180 and 270 min of symptom onset. There were no statistically significant differences between the groups. In the same groups the percentage of those currently smoking

Table 1 The patient characteristics by therapeutic window

\begin{tabular}{llll}
\hline & Golden hour & $61-180 \mathrm{~min}$ & $181-270 \mathrm{~min}$ \\
\hline Number of Patients & 73 & 414 & 126 \\
Median Age & 71 & 72 & 75 \\
Hypertension (\%) & $30(41.1)$ & $194(46.9)$ & $72(57.1)$ \\
Currently Smoking (\%) & $20(27.4)$ & $90(21.7)$ & $19(15.1)$ \\
Atrial Fibrillation (\%) & $11(15.1)$ & $49(11.8)$ & $25(19.8)$ \\
Diabetes (\%) & $3(4.1)$ & $52(12.6)$ & $25(19.8)$ \\
\hline
\end{tabular}

was $27 \%, 21 \%$ and $15 \%$ respectively, again no statistically significant difference was observed. In the same groups the percentages of those with a previously diagnosed atrial fibrillation was $15 \%, 11 \%$ and $19 \%$ respectively - these differences weren't statistically different. In the Golden Hour group only $4 \%$ were diabetics, significantly lower than the $12 \%$ of diabetics in the group treated between 61 and $180 \min (p<0.005)$ and the 19\% of those treated in the final therapeutic window $(p<0.005)$.

Table 2 shows the Clinical Course of the patients treated with IVT in the respective therapeutic windows. The median NIHSS (range) on admission in the six groups was quite similar 7.0 (2-13), 8.0 (2-13), 6.5 (214), 7.0 (2-14), $7.5(2-14)$ and $7.0(2-14)$ - no statistically significant difference was observed. The median NIHSS (range) at discharge in both Golden Hour groups is $0(0-6)$, lower than $4(1-11)$ and $5(2-14)$ in the two subgroups treated between 61 and $180 \mathrm{~min}$ after onset $(p<0.005)$. The median NIHSS at discharge for those treated in the final therapeutic window was 7 in both subgroups, significantly higher than those in the Golden Hour group $(p<0.005)$. The percentage $\mathrm{ICH}$ was $0.0 \%$ in both age subgroups for those treated within the Golden Hour. The percentage $\mathrm{ICH}$ rose to $0.8 \%(p=0.76)$ and $3.9 \%(p=0.44)$ for those treated between 61 and $180 \mathrm{~min}$. The percentage $\mathrm{ICH}$ for those aged $\leq 79$ in the final therapeutic window was $8.0 \%(p=0.02)$ and $19.4 \%(p<0.005)$ for those aged 80 and over.

The in-hospital mortality is $0.0 \%$ for those aged $\leq 79$, treated within the Golden Hour and $7.1 \%$ for those aged 80 and over. The in-hospital mortality for those aged $\leq 79$ treated in the second therapeutic window is $1.5 \%$ and $6.5 \%$ for those 80 and older - no statistically significant difference is observed. In the final therapeutic window, the inhospital mortality for those aged $\leq 79$ is $16.1 \%(p<0.005)$ and $22.3 \%(p<0.005)$ for those 80 and older.

Twenty-five (4.1\%) of our cohort left the hospital with a Stroke Mimic diagnosis; results not shown. 
Table 2 The clinical course of IVT treatment in the different therapeutic windows including age sub-groups

\begin{tabular}{|c|c|c|c|c|c|c|}
\hline & Golden hour & Golden hour & $61-180 \mathrm{~min}$ & $61-180 \mathrm{~min}$ & $181-270 \mathrm{~min}$ & $181-270 \mathrm{~min}$ \\
\hline Age Group & $\leq 79$ & $>80$ & $\leq 79$ & $>80$ & $\leq 79$ & $>80$ \\
\hline Number of Patients & 46 & 27 & 261 & 153 & 62 & 67 \\
\hline Median NIHSS on Admission & 7 & 8 & 6.5 & 7 & 7.5 & 7 \\
\hline Median NIHSS at Discharge & 0 & 0 & 4 & 5 & 7 & 7 \\
\hline $\mathrm{ICH}(\%)$ & $0(0.0)$ & $0(0.0)$ & $2(0.8)$ & $6(3.9)$ & $5(8.0)$ & $13(19.4)$ \\
\hline In-hospital Mortality (\%) & $0(0.0)$ & $2(7.4)$ & $4(1.5)$ & $10(6.5)$ & $10(16.1)$ & $15(22.3)$ \\
\hline
\end{tabular}

\section{Discussion}

Our study showed a significant increase in the number of patients being treated within the Golden Hour as a result of the decreasing DTN - an increase from $2.2 \%$ to $14.5 \%(p=0.006)$. Larger works have shown that less than $29 \%$ of AIS patients actually arrive at the Emergency Room (ER) within $60 \mathrm{~min}$ of onset and of those, only $18 \%$ have a DTN time of $60 \mathrm{~min}$ or less [15]. The benefits of getting to the hospital quickly are often nullified due to the stresses of the ER [16] thus very few patients are actually treated within $60 \mathrm{~min}$ of symptom onset. The fact that our center is consistently treating every eighth patient $(18.2 \%, 14.8 \%, 12.5 \%$ and $14.5 \%)$ within the Golden Hour is quite remarkable.

The benefits of rapid treatment are clear in both age groups, with a median NIHSS of 0 at discharge, significantly better outcomes were achieved in the Golden Hour than in the later therapeutic windows. There was also no incidence of any $\mathrm{ICH}$ for octogenarians and nonagenarians treated with IVT within the Golden Hour. The fact that those aged over 80 achieved such results is of great significance for clinical practice as these patients usually comprise the majority of stroke deaths $[17,18]$. They are usually also excluded from clinical trials and analyses making the decision to treat in the acute phase even more challenging [19].

The in-hospital mortality amongst those $>80$ years old treated in the Golden Hour was also very low (7.4\%). This is noteworthy due to the fact any hospital admission in octogenarians and nonagenarians is associated with significant in-hospital mortality $(20-40 \%)$ [20, 21]. These two cases of in-hospital mortality were both nonagenarians having significant pre-existing comorbidity. Nevertheless, $97.3 \%$ of the octogenarians and nonagenarians had excellent outcomes of IVT therapy within 60 min of symptom onset without any incidence of $\mathrm{ICH}$. With the expected exponential rise in the population of octogenarians and nonagenarians these results provide the firm basis for early treatment in these patients.

The work by Ebinger et al on Golden Hour IVT therapy excludes patients with a stroke mimic diagnosis [12] whereas our work has included these patients. Stroke mimics make up a very real part of patients in the ER. The incidence of mimics in our cohort $(4.1 \%)$ is lower than in other studies [22], and could be due to the fact that mimics tend to complicate the decision to treat and are therefore associated with increased treatment times [23]. Nevertheless, the fact that this patient group can be treated without any $\mathrm{ICH}$ and or poor outcome is of clinical significance in the hyper acute setting. The finding that IVT treatment in stroke mimics is safe is also supported by other works [23, 24].

The retrospective nature of data collection in the earlier years (2009-2011) subjects our study to certain unavoidable weaknesses; however, the majority of patients were prospectively included. The merits of our study include the number of patients included and the number of years of our study. The homogeny of the patient population is also a strength of our study as it excludes any variation in outcomes due to race/ethnicity. The continued and ongoing work at our treatment center toward reducing OTN and DTN times could be a source of bias; the EMS crews and ER staff working more quickly to ensure shorter treatment times for patients with AIS. The bias is somewhat inevitable, but is an important byproduct of our focused work with the members of the treatment chain. We feel that ongoing efforts with the staff involved in the treatment of AIS patients is fundamental to providing rapid and efficacious treatment in the acute setting. The acute setting in the ER can provide unforeseen challenges and stresses, having every member of the team primed toward the goal of rapid treatment is inevitably advantageous.

The excellent outcomes associated with IVT treatment in the Golden Hour in all age groups including stroke mimics suggests that all efforts should be made to ensure treatment within this opportune therapeutic window. These results also reinforce that any efforts made, both pre- and in- hospital, are worthwhile investments in securing the best outcomes for the patient population. The routines surrounding rapid IVT therapy should be extended to elderly patients as they benefit equally from early treatment.

\section{Conclusions}

Acute Ischemic Stroke, it's treatment and thus it's clinical outcomes are time dependent. Significant efforts are being 
made both pre- and in- hospital to provide treatment as quickly as possible. Our results show that treating patients within one hour of symptom onset leads to excellent outcomes, without any incidence of iatrogenic bleeds. These excellent outcomes are also seen in octogenarians, suggesting that age alone should not contradict rapid treatment. The protocols put in place to ensure rapid treatment yield excellent results and should be extended to all patients, irrespective of age.

\section{Acknowledgements}

Linn Rettedal Haraldseid was invaluable in collecting the data for our research database.

\section{Funding}

The study has no funding declarations.

Availability of data and materials

Please contact the author for any data requests.

\section{Authors' contributions}

RA collected the data, analysed the data and drafted the manuscript. HN help draft the manuscript. MK designed the study, helped draft the manuscript and contributed to data analysis. All authors read and approved the final manuscript.

\section{Competing interests}

The authors declare that they have no competing interests.

\section{Consent for publication}

Consent for publication is not applicable to our data/study.

\section{Ethics approval and consent to participate}

The study was performed with the approval of the regional ethics committee (Regional Etisk Komitee - REK).

\section{Publisher's Note}

Springer Nature remains neutral with regard to jurisdictional claims in published maps and institutional affiliations.

\section{Author details}

'Department of Neurology, Stavanger University Hospital, Postboks 8100, Stavanger 4068, Norway. ${ }^{2}$ Neuroscience Research Group, Stavanger University Hospital, Stavanger, Norway. ${ }^{3}$ Department of Neurology, Haukeland University Hospital, Bergen, Norway. ${ }^{4}$ Institute of Clinical Medicine, University of Bergen, Bergen, Norway.

Received: 17 February 2017 Accepted: 12 May 2017

Published online: 22 May 2017

\section{References}

1. Tissue plasminogen activator for acute ischemic stroke. The National Institute of Neurological Disorders and Stroke rt-PA Stroke Study Group. N Engl J Med. 1995;333(24):1581-7.

2. Hacke W, et al. Thrombolysis with alteplase 3 to $4.5 \mathrm{~h}$ after acute ischemic stroke. N Engl J Med. 2008:359(13):1317-29.

3. Hacke W, et al. Randomised double-blind placebo-controlled trial of thrombolytic therapy with intravenous alteplase in acute ischaemic stroke (ECASS II). Second European-Australasian Acute Stroke Study Investigators. Lancet. 1998;352(9136):1245-51.

4. Goyal M, et al. Randomized assessment of rapid endovascular treatment of ischemic stroke. N Engl J Med. 2015;372(11):1019-30.

5. Molina CA, et al. REVASCAT: a randomized trial of revascularization with SOLITAIRE FR device vs. best medical therapy in the treatment of acute stroke due to anterior circulation large vessel occlusion presenting within eight-hours of symptom onset. Int J Stroke. 2015;10(4):619-26.

6. Saver $\mathrm{L}$, et al. Solitaire with the Intention for Thrombectomy as Primary Endovascular Treatment for Acute Ischemic Stroke (SWIFT PRIME) trial: protocol for a randomized, controlled, multicenter study comparing the Solitaire revascularization device with IV IPA with IV IPA alone in acute ischemic stroke. Int J Stroke. 2015;10(3):439-48.

7. Advani $\mathrm{R}$, Naess $\mathrm{H}$, Kurz MW. Evaluation of the implementation of a rapid response treatment protocol for patients with acute onset stroke: can we increase the number of patients treated and shorten the time needed? Cerebrovasc Dis Extra. 2014;4(2):115-21.

8. Meretoja $\mathrm{A}$, et al. Reducing in-hospital delay to $20 \mathrm{~min}$ in stroke thrombolysis. Neurology. 2012;79(4):306-13.

9. Kurz MW, Kurz KD, Farbu E. Acute ischemic stroke-from symptom recognition to thrombolysis. Acta Neurol Scand Suppl. 2013;196:57-64.

10. Hacke W, et al. Dichotomized efficacy end points and global end-point analysis applied to the ECASS intention-to-treat data set: post hoc analysis of ECASS I. Stroke. 1998;29(10):2073-5.

11. John S, et al. Performance of CT Angiography on a Mobile Stroke Treatment Unit: Implications for Triage. 2016. J Neuroimaging.

12. Ebinger $\mathrm{M}$, et al. Effects of golden hour thrombolysis: a Prehospital Acute Neurological Treatment and Optimization of Medical Care in Stroke (PHANTOM-S) substudy. JAMA Neurol. 2015;72(1):25-30.

13. Warach S. Prehospital thrombolysis for stroke: an idea whose golden hour has arrived. JAMA Neurol. 2015;72(1):9-10.

14. Advani R, Naess H, Kurz M. Mass Media Intervention in Western Norway Aimed at Improving Public Recognition of Stroke, Emergency Response, and Acute Treatment. J Stroke Cerebrovasc Dis. 2016;25(6):1467-72.

15. Saver $\mathrm{JL}$, et al. The "golden hour" and acute brain ischemia: presenting features and lytic therapy in $>30,000$ patients arriving within 60 min of stroke onset. Stroke. 2010;41(7):1431-9.

16. Strbian D, et al. Relationship between onset-to-door time and door-tothrombolysis time: a pooled analysis of 10 dedicated stroke centers. Stroke. 2013:44(10):2808-13.

17. Mateen FJ, et al. Outcomes of thrombolysis for acute ischemic stroke in octogenarians versus nonagenarians. Stroke. 2010;41(8):1833-5.

18. Elkins JS, Johnston SC. Thirty-year projections for deaths from ischemic stroke in the United States. Stroke. 2003;34(9):2109-12.

19. Wardlaw JM, et al. Thrombolysis for acute ischaemic stroke. Cochrane Database Syst Rev. 2009;4, CD000213.

20. Garrouste-Orgeas M, et al. Treatment intensity and outcome of nonagenarians selected for admission in ICUs: a multicenter study of the Outcomerea Research Group. Ann Intensive Care. 2016;6(1):31.

21. Iwamoto T, et al. Effectiveness of Hospital Functions for Acute Ischemic Stroke Treatment on In-Hospital Mortality: Results From a Nationwide Survey in Japan. J Epidemiol. 2015;25(8):522-8.

22. Gargalas $\mathrm{S}$, et al. Incidence and outcome of functional stroke mimics admitted to a hyperacute stroke unit. 2015. J Neurol Neurosurg Psychiatry.

23. Sivakumaran P, et al. A Retrospective Cohort Study on the Use of Intravenous Thrombolysis in Stroke Mimics. J Stroke Cerebrovasc Dis. 2016; 25(5):1057-61.

24. Tsivgoulis G, et al. Safety of intravenous thrombolysis in stroke mimics: prospective 5-year study and comprehensive meta-analysis. Stroke. 2015; 46(5):1281-7.

Submit your next manuscript to BioMed Central and we will help you at every step:

- We accept pre-submission inquiries

- Our selector tool helps you to find the most relevant journal

- We provide round the clock customer support

- Convenient online submission

- Thorough peer review

- Inclusion in PubMed and all major indexing services

- Maximum visibility for your research

Submit your manuscript at www.biomedcentral.com/submit
C Biomed Central 